\title{
Exploring predictors of surgery and comparing operative treatment approaches for pediatric intracranial arachnoid cysts: a case series of 83 patients
}

\author{
Mohsin Ali, MPhil, ${ }^{1}$ Michael Bennardo, MSc, ${ }^{2}$ Saleh A. Almenawer, MD, ${ }^{2}$ Nirmeen Zagzoog, MD, ${ }^{2}$ \\ Alston A. Smith, BSc, ${ }^{3}$ Dyda Dao, BHSc, ${ }^{3}$ Olufemi Ajani, MD, ${ }^{2}$ Forough Farrokhyar, MPhil, PhD, ${ }^{3,4}$ \\ and Sheila K. Singh, MD, PhD² \\ ${ }^{1}$ Icahn School of Medicine at Mount Sinai, New York, New York; ${ }^{2}$ Division of Neurosurgery, Departments of ${ }^{3}$ Surgery and ${ }^{4} \mathrm{Clinical}$ \\ Epidemiology and Biostatistics, McMaster University, Hamilton, Ontario, Canada
}

\begin{abstract}
OBJECT Although intracranial arachnoid cysts are a common incidental finding on pediatric brain imaging, only a subset of patients require surgery for them. For the minority who undergo surgery, the comparative effectiveness of various surgical approaches is debated. The authors explored predictors of surgery and compared operative techniques for pediatric patients with an intracranial arachnoid cyst seen at a tertiary care center.

METHODS The authors reviewed records of pediatric patients with an intracranial arachnoid cyst. For each patient, data on baseline characteristics, the method of intervention, and surgical outcomes for the initial surgery were extracted, and cyst size at diagnosis was calculated (anteroposterior $\times$ craniocaudal $\times$ mediolateral). Baseline variables were analyzed as predictors of surgery by using logistic regression modeling, excluding patients whose surgery was not related to cyst size (i.e., those with obstructive hydrocephalus secondary to the cyst compressing a narrow CSF flow pathway or cyst rupture/hemorrhage). Data collected regarding surgical outcomes were analyzed descriptively.
\end{abstract}

RESULTS Among 83 pediatric patients with an intracranial arachnoid cyst seen over a 25-year period (1989-2013), 27 (33\%) underwent surgery; all had at least 1 cyst-attributed symptom/finding. In the multivariate model, age at presentation and cyst size at diagnosis were independent predictors of surgery. Cyst size had greater predictive value; specifically, the area under the curve for the receiver-operating-characteristic curve was $0.89(95 \% \mathrm{Cl} 0.82-0.97)$, with an ideal cutoff point of $\geq 68 \mathrm{~cm}^{3}$. This cutoff point had $100 \%$ sensitivity (95\% Cl 79\%-100\%), $75 \%$ specificity $(95 \% \mathrm{Cl} 61 \%-85 \%)$, a $53 \%$ positive predictive value $(95 \% \mathrm{Cl} 36 \%-70 \%)$, and a $100 \%$ negative predictive value $(95 \% \mathrm{Cl} 91 \%-100 \%)$; the positive likelihood ratio was $4.0(95 \% \mathrm{Cl} 2.5-6.3)$, and the negative likelihood ratio was $0(95 \% \mathrm{Cl} 0-0.3)$. Although the multivariate model excluded 7 patients who underwent surgery (based on prespecified criteria), excluding these 7 cases did not change the overall findings, as shown in a sensitivity analysis that included all the cases. Descriptive results regarding surgical outcomes did not indicate any salient differences among the surgical techniques (endoscopic fenestration, cystoperitoneal shunting, or craniotomy-based procedures) in terms of symptom resolution within 6 months, need for reoperation to date, cyst-size change from before the operation, morbidity, or mortality.

CONCLUSIONS The results of these exploratory analyses suggest that pediatric patients with an intracranial arachnoid cyst are more likely to undergo surgery if the cyst is large, compresses a narrow CSF flow pathway to cause hydrocephalus, or has ruptured/hemorrhaged. There were no salient differences among the 3 surgical techniques for several clinically important outcomes. A prospective multicenter study is required to enable more robust analyses, which could ultimately provide a decision-making framework for surgical indications and clarify any differences in the comparative effectiveness of surgical approaches to treating pediatric intracranial arachnoid cysts.

http://thejns.org/doi/abs/10.3171/2015.2.PEDS14612

KEY WORDS arachnoid cysts; craniotomy; cystoperitoneal shunt; endoscopic fenestration; predictors; surgery; congenital

ABBREVIATIONS AUC = area under the curve; $\mathrm{CP}=$ cystoperitoneal; $I Q R=$ interquartile range; $\mathrm{ROC}=$ receiver operating characteristic

SUBMITTED November 6, 2014. ACCEPTED February 19, 2015.

INCLUDE WHEN CITING Published online June 12, 2015; DOI: 10.3171/2015.2.PEDS14612.

DISCLOSURE The authors report no conflict of interest concerning the materials or methods used in this study or the findings specified in this paper. Dr. Singh is supported by the Canada Research Chairs program. 
I NTRACRANIAL arachnoid cysts are collections of CSF within the subarachnoid space of the meninges, which may become contained when the arachnoid membrane forms septations..$^{15}$ These lesions are common among the pediatric population and are most often congenital, developing during the cortical migration phase of fetal development, although acquired cysts have been described..$^{8,13}$

The reported prevalence of pediatric intracranial arachnoid cysts has increased with the growing use of intracranial imaging, particularly CT and MRI. A recent estimate for prevalence among 11,738 patients aged $\leq 18$ years who underwent MRI at a single institution over an 11-year period was $2.6 \%$ for intracranial arachnoid cysts. ${ }^{1}$ The majority of these lesions were incidental and asymptomatic, observations that are consistent with those in previous research. ${ }^{11,12,18}$

When children and adolescents with an intracranial arachnoid cyst become symptomatic, it is often due to cyst growth, the underlying mechanisms of which are unknown. Several mechanisms have been proposed, including active fluid secretion by cells in the cyst wall, expansion after formation of an osmotic gradient, and unidirectional valve mechanisms., ${ }^{4,16,19}$ Cyst growth can lead to clinically important symptoms through increased intracranial pressure and/or compression of surrounding brain structures, including a CSF flow pathway. Symptoms can also arise suddenly after cyst rupture or hemorrhage into a cyst, and for both cases (cyst growth and cyst rupture/ hemorrhage), symptom type and severity tend to depend on cyst location. ${ }^{19}$

Most children and adolescents with an intracranial arachnoid cyst will remain asymptomatic and will not require surgery. ${ }^{1}$ Identifying predictors for surgery, therefore, can inform decision-making about clinical management. There is a paucity of literature addressing the predictors of surgery among these patients, with only 1 recent study suggesting that larger cyst size at diagnosis and a younger age at presentation are statistically significant predictors. ${ }^{1}$ In addition, for the minority of children and adolescents with an intracranial arachnoid cyst who undergo surgery, the comparative effectiveness of various surgical approaches for treating pediatric intracranial arachnoid cysts (e.g., endoscopic fenestration, cystoperitoneal [CP] shunting, and craniotomy-based procedures) is debated. ${ }^{3,10,14,17,20}$ Given these areas of uncertainty, we undertook a case-series study among patients referred to our pediatric neurosurgery practice, which is based at a tertiary care center. The primary objectives were to explore predictors of surgery and compare outcomes among the surgical techniques for pediatric intracranial arachnoid cysts.

\section{Methods \\ Data Collection}

We retrospectively reviewed all records (electronic and paper) of patients aged $\leq 18$ years with a radiologically confirmed intracranial arachnoid cyst, as identified in August 2011 using the electronic medical record system at McMaster Children's Hospital in Hamilton, Ontario, Canada. To achieve high search sensitivity, we broadly queried the system with the terms "brain" and "cyst" and subsequently reviewed all records found to identify patients with an intracranial arachnoid cyst. In addition, from $\mathrm{Au}-$ gust 2011 through September 2013, eligible patients were prospectively identified, because ours is the only pediatric neurosurgical practice in the region.

We reviewed all neurosurgical records for all the identified patients. Demographic data, including age at presentation and sex, were extracted for each patient. The following clinical and radiological data were also extracted: cyst-attributed symptoms and findings (including hydrocephalus and cyst growth), cyst location, and, when available, cyst size at diagnosis and at the most recent followup visit (based on CT or MRI). Cyst growth was defined, as reported by the radiologist, as an increase in cyst size between the initial CT/MR images and subsequent CT/ MR images (acquired by using the same imaging modality per patient). Cyst size was recorded as the reported maximal anteroposterior, craniocaudal, and mediolateral dimensions (in centimeters) and was calculated in terms of volume (in cubic centimeters) by multiplying the reported dimensions (i.e., anteroposterior $\times$ craniocaudal $\times$ mediolateral). For patients with a reoperation, cyst size at follow-up corresponded to the most recent size noted before the second surgery.

Throughout the study period, the decision to operate and the specific surgical technique used were based on surgeon preference. For patients who underwent surgery, data were extracted for only the first arachnoid cyst-related surgery. These data included the surgical technique used and the following surgical outcomes: symptom resolution within 6 months, need for reoperation to date, length of stay in hospital, cyst-size change from before the operation, morbidity (CSF leak, infection, and/or hemorrhage), and mortality.

The study protocol was approved by the Hamilton Health Sciences/Faculty of Health Sciences research ethics board at McMaster University in Hamilton, Ontario, Canada.

\section{Statistical Analysis}

Baseline characteristics (i.e., sex, age at presentation, cyst size at diagnosis, and cyst location) and number of cyst-attributed symptoms/findings were summarized for patients managed conservatively and for those managed surgically. Categorical variables were reported as frequencies or relative frequencies and compared by using the Pearson chi-square test or Fisher's exact test, as applicable; the Monte Carlo test was used when the expected counts were $<5$. Continuous variables were reported as means with the SD or, if not normally distributed, as medians with the interquartile range (IQR) and compared by using the Student t-test or Mann-Whitney U-test, respectively.

To explore predictors of surgery, baseline variables with a $\mathrm{p}$ value of $<0.1$ in univariate analyses (i.e., age at presentation and cyst size at diagnosis) were entered into a multivariate stepwise logistic regression model. We used 10 patients (i.e., those who underwent surgery) and 10 controls (i.e., those who did not undergo surgery) per parameter, as a rule of thumb regarding statistical power and fit for multivariate logistic regression modeling. ${ }^{9}$ The 
specific or overall numbers of cyst-attributed symptoms/ findings were not considered baseline variables, because neither all symptoms nor findings (e.g., cyst growth) had developed at diagnosis. The multivariate analysis excluded the small minority of patients whose surgery was not related to cyst size, namely, those who experienced sudden symptoms as a result of cyst rupture/hemorrhage or had obstructive hydrocephalus secondary to the cyst growing to compress a narrow CSF flow pathway (e.g., cerebral aqueduct, foramina of Monro). In such cases, larger cyst sizes (in absolute terms) are not related to the need for surgery and thus would have diminished the predictive value. However, to investigate whether these exclusions changed the overall findings, we conducted a sensitivity analysis that included all cases in the multivariate model. For all regression analyses, odds ratios with $95 \%$ confidence intervals are reported; model fit was assessed by using the Hosmer-Lemeshow goodness-of-fit test.

We plotted a receiver-operating-characteristic (ROC) curve based on the optimal predictor, evaluated by the area-under-the-curve (AUC) statistic. The optimal cutoff (in terms of sensitivity and specificity) was calculated from the ROC curve.

Data collected for 2 other areas of importance regarding the clinical and surgical management of pediatric intracranial arachnoid cysts were analyzed descriptively because of the small sample size. These areas were 1) reasons why patients who were initially followed conservatively eventually underwent surgery and 2) surgical outcomes for all patients and per surgical technique (endoscopic fenestration, $\mathrm{CP}$ shunting, and craniotomy-based procedures).

All statistical tests were 2-sided, and a p value of $<0.05$ was considered statistically significant. Stata 13.1 (StataCorp.) was used for data analysis.

\section{Results}

Among 337 records retrospectively reviewed (earliest from February 1989) and eligible cases included prospectively through September 2013, 83 pediatric patients with an intracranial arachnoid cyst were identified. MRI was available at our institution throughout this 25-year period. Twenty-seven (33\%) patients underwent surgery, all of whom had at least 1 cyst-attributed symptom or finding (Fig. 1); the most common cyst-attributed symptoms/ findings among these patients were headache/irritability (44\%), macrocephaly (37\%), and cyst growth (37\%). The median age at presentation for patients with cyst growth was < 1 month (IQR 20 months); the oldest patient was 46 months old. All but 1 patient managed conservatively had no cyst-attributed symptom/finding; the exceptional patient had macrocephaly.

Sixty-two $(75 \%)$ patients were male. In terms of cyst location, $46(55 \%)$ patients had a cyst in the middle fossa, and $18(22 \%)$ had a cyst in the posterior fossa. In univariate analyses among the children managed conservatively versus those managed surgically, the age at presentation (median \pm IQR $87 \pm 101$ vs $18 \pm 67$ months, respectively; $\mathrm{p}=0.002$ ), cyst size at diagnosis (median $\pm \mathrm{IQR} 26 \pm 63$ vs $92 \pm 439 \mathrm{~cm}^{3}$, respectively; $\mathrm{p}<0.001$ ), and proportion of patients with $\geq 1$ cyst-attributed symptom/finding $(2 \%$ vs $100 \%$, respectively; $\mathrm{p}<0.001)$ were statistically signifi-

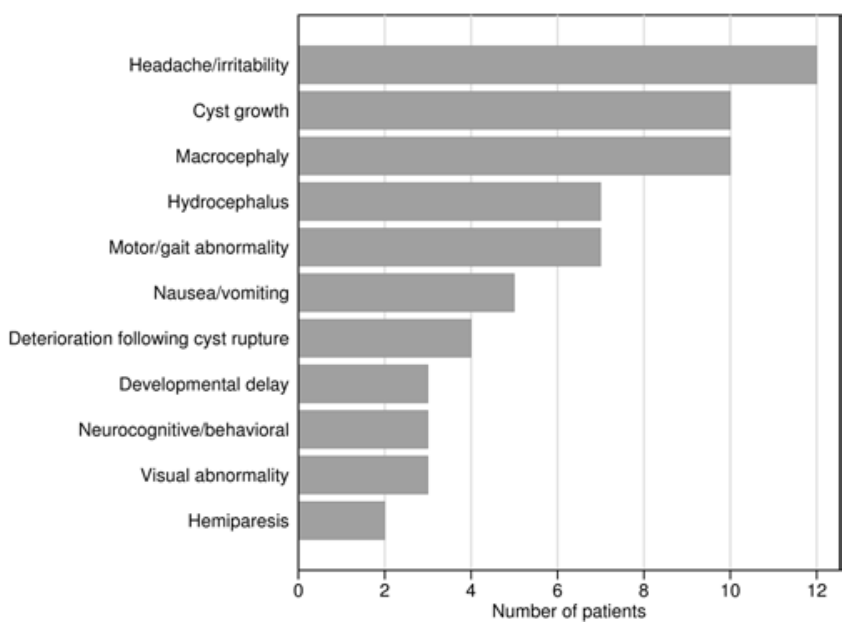

FIG. 1. Individual breakdown of cyst-attributed symptoms and findings for 27 patients who underwent surgery (among 83 pediatric patients with an intracranial arachnoid cyst). None of the patients had seizures or endocrinopathy.

cantly different; sex (77\% vs 70\% male, respectively; $\mathrm{p}=$ 0.59 ) and cyst location were not (Table 1).

In a multivariate analysis, age at presentation and cyst size at diagnosis were independent and statistically significant predictors of surgery without interaction $(\mathrm{p}$ $=0.71)$. Specifically, the OR was 0.984 per month $(95 \%$ CI 0.971-0.998 per month; $p=0.02$ ) for age at presentation and 1.008 per $\mathrm{cm}^{3}$ (95\% CI 1.003-1.012 per $\mathrm{cm}^{3} ; \mathrm{p}=$ $0.001)$ for cyst size at diagnosis. Results of the HosmerLemeshow goodness-of-fit test indicated adequate model fit $(p=0.58)$. Seven patients who underwent surgery were excluded from the multivariate model based on prespecified criteria: 5 patients had obstructive hydrocephalus related to an intracranial arachnoid cyst compressing a narrow CSF flow pathway (cerebral aqueduct [3 patients], foramina of Monro [2 patients]), and 2 patients experienced sudden clinical deterioration after cyst rupture (1 patient had a recent history of head trauma). Excluding these 7 cases did not change our overall findings; in a sensitivity analysis that included all cases, age at presentation and cyst size at diagnosis remained independent and statistically significant predictors of surgery without interaction (Table 2). In addition, surgery for 3 other patients (2 with hydrocephalus and 1 with sudden deterioration after cyst rupture) was indicated by large cyst size; thus, these cases were included in the multivariate analysis per prespecified criteria.

Cyst size at diagnosis was the most optimal predictor of surgery (AUC 0.89; 95\% CI 0.82-0.97) (Fig. 2). The ideal cutoff point was $\geq 68 \mathrm{~cm}^{3}$, which had $100 \%$ sensitivity (95\% CI 79\%-100\%), 75\% specificity (95\% CI 61\%-85\%), a $53 \%$ positive predictive value (95\% CI 36\%-70\%), and a $100 \%$ negative predictive value (95\% CI 91\%-100\%), corresponding to a positive likelihood ratio of $4.0(95 \%$ CI 2.5-6.3) and a negative likelihood ratio of 0 (95\% CI $0-0.3)$. Recent MR images of 4 patients are shown in Fig. 3 to provide a visual depiction of large (Fig. 3A and B) and small (Fig. 3C and D) cysts relative to this $68-\mathrm{cm}^{3}$ cutoff point.

Five patients who were initially managed conserva- 
TABLE 1. Univariate analyses between patients managed conservatively and those managed surgically

\begin{tabular}{|c|c|c|c|}
\hline Patient or Cyst Characteristic & Conservative Treatment $(n=56)$ & Surgery $(n=27)$ & $p$ Value* $^{*}$ \\
\hline Male (no. [\%]) & $43(77)$ & $19(70)$ & 0.59 \\
\hline Age at presentation, mos (median [IQR]) & $87(101)$ & $18(67)$ & 0.002 \\
\hline Cyst size at diagnosis, $\mathrm{cm}^{3}$ (median [IQR])† & $26(63)$ & $92(439)$ & $<0.0001$ \\
\hline No. $(\%)$ w/ $\geq 1$ cyst-attributed symptom & $1(2)$ & $27(100)$ & $<0.0001$ \\
\hline \multicolumn{4}{|l|}{ Cyst location (no. [\%])‡ } \\
\hline Middle fossa & $34(74)$ & $12(26)$ & 0.24 \\
\hline Posterior fossa & $13(72)$ & $5(28)$ & 0.78 \\
\hline Intraventricular & $3(60)$ & $2(40)$ & 0.66 \\
\hline Interhemispheric & $3(60)$ & $2(40)$ & 0.66 \\
\hline Quadrigeminal plate & $3(60)$ & $2(40)$ & 0.66 \\
\hline Sellar/suprasellar & $0(0)$ & $3(100)$ & 0.06 \\
\hline Cerebral convexity & $0(0)$ & $1(100)$ & 0.33 \\
\hline \multicolumn{4}{|c|}{$\begin{array}{l}\text { * Based on Pearson's chi-square statistic, Mann-Whitney U-test, and Fisher's exact test, as applicable. The Monte Carlo test was used wher } \\
\text { expected counts were }<5 \text {. } \\
\dagger \text { Cyst size was calculated by multiplying reported dimensions (anteroposterior } \times \text { craniocaudal } \times \text { mediolateral). For } 4 \text { cases in the surgical } \\
\text { group, however, neither these data nor corresponding MR/CT images were accessible. } \\
\ddagger \text { The denominator for each of these proportions is all patients (conservatively or surgically managed) with a cyst in that location. }\end{array}$} \\
\hline
\end{tabular}

tively and eventually underwent surgery are characterized descriptively in Table 3. For 3 cases, the primary indication for surgery was cyst growth, as noted in serial MRI.

Surgical follow-up and outcomes of all 27 patients and according to 3 common surgical techniques for intracranial arachnoid cysts are summarized descriptively in Table 4. The median follow-up time among the surgical techniques was $>1$ year. All but 1 patient were followed up for $>6$ months; the exceptional case was followed up for only 1 month (for this study) because his surgery occurred 1 month before the end of the study period. Eighteen (67\%) patients experienced symptom resolution within 6 months; most of them were children who had a CP shunt $(8$ of 10 [80\%] patients). However, $11(41 \%)$ patients required at least 1 reoperation of their initial surgery, of whom 6 (22\%) have required $>1$ reoperation to date. Reoperations were also most common in children with $\mathrm{CP}$ shunts (5 of 10 [50\%] patients), 3 (60\%) of which were revised as a result of shunt malfunctioning.

The most common surgical morbidity was CSF leak, which occurred in $5(19 \%)$ patients, 3 of whom had a craniotomy-based procedure. Three (11\%) patients had a postoperative infection; 1 patient had a $\mathrm{CP}$ shunt, another had an endoscopic fenestration that was accompanied by a CSF leak, and 1 underwent bur hole drainage. Finally, 1 patient experienced a mild asymptomatic hemorrhage (chronic subdural hematoma) after endoscopic fenestration, ostensibly because a decrease in intracranial pressure after a rapid cyst-size decrease (from $192 \mathrm{~cm}^{3}$ to $57 \mathrm{~cm}^{3}$ within 1 month) caused bridging subdural veins to rupture. No deaths occurred.

In terms of cyst-size change from before the operation, $18(67 \%)$ patients' cysts decreased in size (by more than $10 \mathrm{~cm}^{3}$ ) and $6(22 \%)$ patients' cysts increased in size (by more than $\left.10 \mathrm{~cm}^{3}\right)$, whereas $3(11 \%)$ patients' cysts were unchanged (within $10 \mathrm{~cm}^{3}$ ). Four of the 6 patients whose cyst had grown postoperatively were aged $\leq 2$ years at presentation; 2 of 6 (both of whom were aged $\leq 2$ years at presentation) underwent reoperation during the study period, 1 related to $\mathrm{CP}$ shunt malfunction and the other related to closure of fenestration. Cyst-size change was not associated with postoperative symptom resolution within 6 months ( $p=0.67$; data not shown). The median length of hospital stay was 5 days (IQR 3 days); the patients who underwent endoscopic fenestration stayed for the shortest duration on average (median \pm IQR $3 \pm 1$ days).

TABLE 2. Sensitivity analysis of multivariate logistic regression models for predictors of surgery*

\begin{tabular}{cccc}
\hline Model \& Predictors & OR $(95 \% \mathrm{Cl})$ & p Value & AUC $(95 \% \mathrm{Cl}) \dagger$ \\
\hline All cases $(\mathrm{n}=79)$ & & & \\
\hline Age at presentation $(\mathrm{mos})$ & $0.982(0.971-0.994)$ & 0.003 & $0.73(0.61-0.86)$ \\
\hline Cyst size at diagnosis $\left(\mathrm{cm}^{3}\right)$ & $1.006(1.002-1.010)$ & 0.004 & $0.80(0.69-0.91)$ \\
\hline All ops related to cyst size $(\mathrm{n}=72)$ & & & \\
\hline Age at presentation $(\mathrm{mos})$ & $0.984(0.971-0.998)$ & 0.02 & $0.72(0.58-0.86)$ \\
\hline Cyst size at diagnosis $\left(\mathrm{cm}^{3}\right)$ & $1.008(1.003-1.012)$ & 0.001 & $0.89(0.82-0.97)$ \\
\hline
\end{tabular}

\footnotetext{
* Model fit, as assessed by using the Hosmer-Lemeshow goodness-of-fit test, was adequate for both models-for all cases $(p=0.38)$ and for surgeries related to cyst size $(p=0.58)$. Interactions between the predictors were nonsignificant $(p=0.82$ for all cases, $p=0.71$ for surgeries related to cyst size) and therefore were not included in either model.

$\dagger$ The AUC reported pertains to only the predictor of interest rather than to a model with multiple predictors.
} 


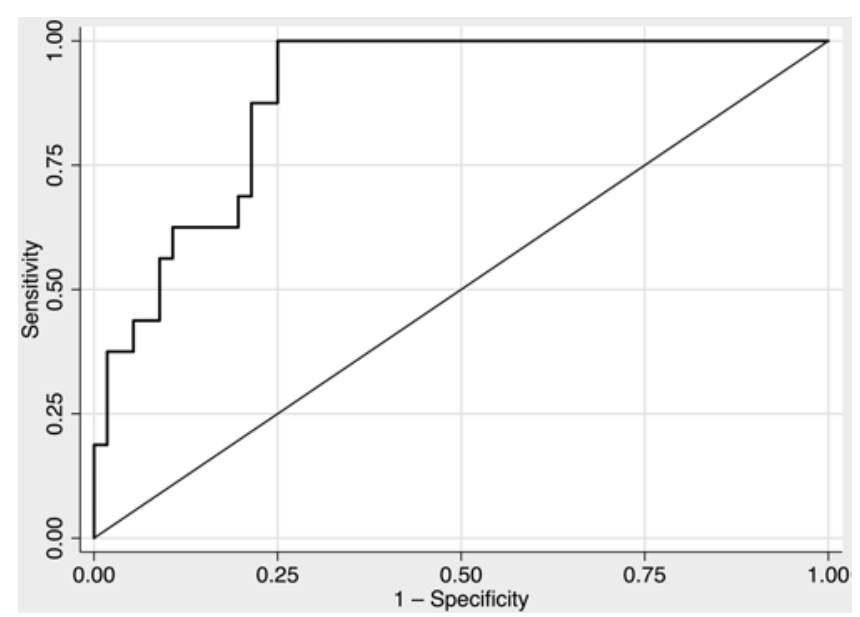

FIG. 2. ROC curve of cyst size at diagnosis as a predictor of surgery $(\mathrm{n}=$ 72; AUC 0.89; 95\% Cl 0.82-0.97).

\section{Discussion}

In this study, conducted at the sole regional pediatric neurosurgery center at a tertiary children's hospital, we identified 83 patients with intracranial arachnoid cysts seen over a 25-year period (February 1989 to September 2013), 27 (33\%) of whom underwent surgery. Each patient who underwent surgery had at least 1 cyst-attributed symptom/finding, whereas none of the patients managed conservatively (except for 1 patient with macrocephaly) had cyst-attributed symptoms/findings. The number of cyst-attributed symptoms/findings, age at presentation, and cyst size at diagnosis were statistically significant in univariate analyses among patients managed conservatively versus those who were managed surgically, findings which are concordant with those of a previous study. ${ }^{1}$ However, in contrast to previous research that found positive correlations of anterior fossa and quadrigeminal plate cysts and an inverse correlation of posterior fossa cysts to be predictors of surgery, ${ }^{1}$ cyst location was not statistically significant in our analyses, which may be a result of our relatively smaller sample size.

In a multivariate exploratory analysis, we also found that both age at presentation and cyst size at diagnosis were independent predictors of surgery. Of these predictors, cyst size at diagnosis had greater predictive value in terms of the AUC.

Seven patients who underwent surgery were excluded from the multivariate model based on prespecified criteria: 5 patients had obstructive hydrocephalus related to an intracranial arachnoid cyst compressing a narrow CSF flow pathway (cerebral aqueduct [3 patients], foramina of Monro [2 patients]), and 2 patients experienced sudden clinical deterioration after cyst rupture (1 patient had a recent history of head trauma). In addition, surgery for 3 other patients ( 2 with hydrocephalus and 1 with sudden deterioration after cyst rupture) was indicated by large cyst size, and thus their cases were included in the multivariate analysis per the prespecified criteria. We believe that excluding the patients whose surgery was not related to cyst size is a clinically appropriate mode of analysis, because although surgery for patients with an intracranial arachnoid cyst is often prompted by clinical symptoms (presumably because of a relatively large cyst that causes mass effect or raises intracranial pressure), in the minority of cases sudden symptoms can arise with cyst rupture/ hemorrhage or obstructive hydrocephalus caused by a cyst growing to compress a narrow CSF flow pathway (e.g., cerebral aqueduct, foramina of Monro). In such cases, a larger cyst size (in absolute terms) is not related to the need for surgery and thus would have diminished the predictive value. Nevertheless, we conducted a sensitivity analysis that included all cases, which showed that excluding these 7 cases did not change our overall findings: both age at presentation and cyst size at diagnosis remained independent and statistically significant predictors of surgery, and cyst size at diagnosis had greater predictive value in terms of the AUC.

Overall, our findings suggest that among children and adolescents with an intracranial arachnoid cyst, 3 groups of patients are more likely to undergo surgery, namely, those who have a cyst that 1 ) is significantly large (e.g., $\geq 68 \mathrm{~cm}^{3}$ based on our analyses), 2) compresses a narrow CSF flow pathway (e.g., cerebral aqueduct, foramina of Monro), or 3) has ruptured/hemorrhaged, as noted in a previous study in which approximately $70 \%$ of pediatric patients with a ruptured/hemorrhaged arachnoid cyst underwent surgery. ${ }^{6}$ Risk factors for pediatric arachnoid cyst rupture/hemorrhage were investigated recently in a matched case-control study of 14 cases, in which larger cyst size (OR 16.5; 95\%

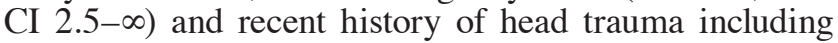
minor falls (OR 25.1; 95\% CI 4.0- $\infty$ ) were identified as risk factors; altitude of residence was not associated with arachnoid cyst rupture or hemorrhage. ${ }^{6}$ Altogether, these findings suggest the potential value of using cyst size at diagnosis as a predictor of surgery, which would be convenient for clinical decision-making, because intracranial arachnoid cysts are almost always diagnosed by using CT or MRI.

Furthermore, we identified 5 patients who were initially conservatively managed but eventually underwent surgery. For 3 of these patients, surgery was prompted by significant cyst growth, as noted on serial MRI. It is interesting to note that these 3 patients (all $\leq 2$ weeks old) were younger by far than the other 2 patients (28 months and 10 years old), which aligns with the understanding that cyst growth is more likely to occur in younger children, particularly those aged $<4$ years. ${ }^{1}$ This finding suggests value in the use of serial MRI for young children with an intracranial arachnoid cyst. However, given the limited data, this topic requires additional research, including study of whether there is an age range for which serial MRI is especially valuable for incidentally found pediatric intracranial arachnoid cysts.

Finally, our descriptive results regarding surgical outcomes do not suggest any salient differences among the surgical techniques (endoscopic fenestration, CP shunting, or craniotomy-based procedures). Our rates of surgical revision after endoscopic fenestration (3 of 7 [43\%]) and CP shunting (5 of 10 [50\%]) are similar to those of other single-center case series of patients with an arachnoid cyst. ${ }^{2,5}$ Furthermore, although $6(22 \%)$ patients had cyst growth $\left(>10 \mathrm{~cm}^{3}\right)$ compared with before the operation, 4 

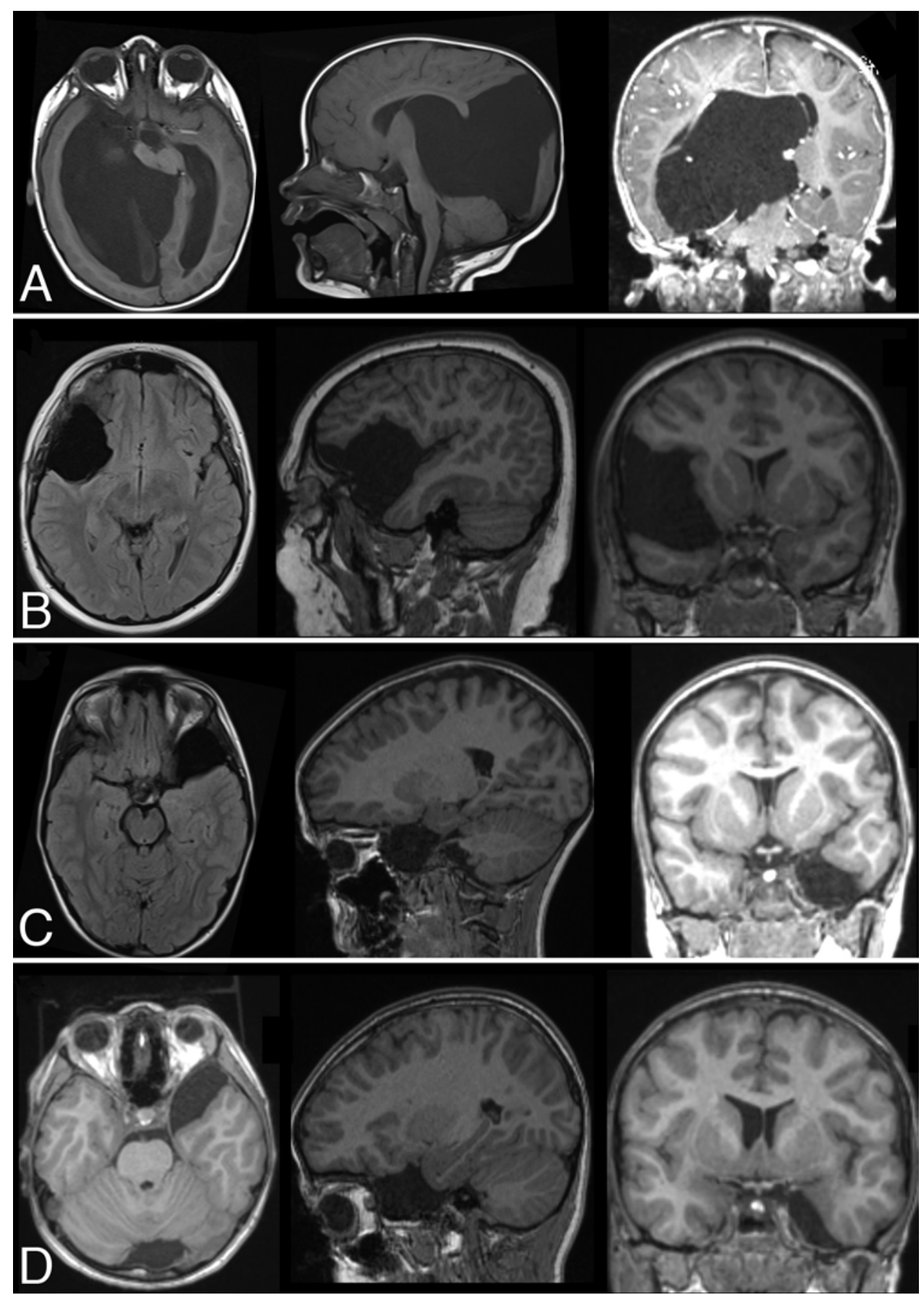

FIG. 3. Axial, sagittal, and coronal T1-weighted MR images (without gadolinium enhancement, except for the coronal image in A) of 4 patients with an intracranial arachnoid cyst. $A$ and $B$ : Two patients with a calculated cyst size (anteroposterior $\times$ craniocaudal $\times$ mediolateral) of $>68 \mathrm{~cm}^{3}$, a 17-month-old boy (A) $\left(960 \mathrm{~cm}^{3}\right)$ and a 13-year-old boy (B) $\left(192 \mathrm{~cm}^{3}\right)$. Both patients underwent surgery, given their cyst-attributed symptoms/findings. C and D: Two patients with a calculated cyst size of $<68 \mathrm{~cm}^{3}$, a 7 -year-old girl (C) $\left(64 \mathrm{~cm}^{3}\right)$ and a 9-year-old boy (D) $\left(46 \mathrm{~cm}^{3}\right)$. Both patients were asymptomatic and have not undergone surgery to date.

of 6 were aged $\leq 2$ years at presentation, an age range in which most head growth occurs (thereby suggesting that the cyst may have enlarged proportionally with the child's growth). In addition, 2 of 6 patients (both of whom were aged $\leq 2$ years at presentation) underwent reoperation, 1 related to $\mathrm{CP}$ shunt malfunction and the other related to closure of fenestration, which may also partially explain the postoperative cyst enlargement. In terms of surgical techniques, since 1989 (the beginning of our study period), institutions have increasingly moved toward neuroendoscopic approaches for arachnoid cysts. This case series was situated within that period of change, with a solo pediatric neurosurgeon at our institution until 2007 who preferred CP shunting over endoscopic fenestration; hence, a substantial proportion (37\% [10 of 27]) of our patients underwent $\mathrm{CP}$ shunting. Nevertheless, equipoise 
TABLE 3. Reasons why patients who were initially followed conservatively eventually underwent surgery

\begin{tabular}{cccccc}
\hline $\begin{array}{c}\text { Patient } \\
\text { No. }\end{array}$ & Sex & $\begin{array}{c}\text { Age at } \\
\text { Presentation }\end{array}$ & Cyst Location & $\begin{array}{c}\text { Age at } \\
\text { Surgery }\end{array}$ & Primary Indication(s) for Surgery \\
\hline 1 & M & 6 days & Interhemispheric & 8 mos & $\begin{array}{c}\text { Cyst growth noted on serial MRI w/ interval macrocephaly (50th- } \\
98 \text { th percentile) }\end{array}$ \\
\hline 2 & F & 2 wks & Lt posterior fossa & 8 mos & $\begin{array}{c}\text { Cyst growth noted on serial MRI w/ subsequent interference to CSF } \\
\text { flow }\end{array}$ \\
\hline 3 & M & 2 wks & Suprasellar & 16 mos & $\begin{array}{c}\text { Cyst growth noted on serial MRI } \\
\text { M }\end{array}$ \\
\hline 5 & M & 10 yrs & Rt temporal fossa & 13 yrs & Worsening headaches \& gait abnormalities \\
\hline
\end{tabular}

continues to persist regarding the comparative effectiveness of surgical approaches for treating pediatric intracranial arachnoid cysts, with some studies ascribing benefit to a particular approach (e.g., endoscopic fenestration, CP shunting, craniotomy-based procedures) ${ }^{10,14,17,20}$ and other research describing comparable benefits. ${ }^{3}$ This area of inquiry also requires additional study.

Our study has several limitations. Because this study was based at a neurosurgical practice, in which the prevalence of surgery-requiring arachnoid cysts is higher than that in the primary care setting, referral bias likely influenced our estimates for predictors of surgery. Thus, we were unable to adjust for factors that primary care practitioners may have accounted for when considering neurosurgical referral for these patients. This may also partially explain the high rate of operation (33\% [27 of 83]), because we studied a highly selected group of patientsthose referred by primary care practitioners because of a clinical/radiological abnormality. Furthermore, this study was mostly retrospective and thus prone to bias and missing data, the latter of which would have not only lead to underestimation of the total number of cases seen during the study period but also may have contributed to the high rate of operation, given that patients who have had an operation are more likely to be found in a retrospective chart review. This was also a single-center study, thereby resulting in a relatively small sample size and limiting its generalizability. Finally, throughout the study period, the decision to operate and the specific surgical technique used were based on surgeon preference rather than being randomized or performed by using a common protocol. Therefore, our regression analyses of predictors of surgery should be regarded as exploratory and for hypothesis generation. Although it is one of the largest studies of pediatric intracranial arachnoid cysts to date, we also did not have sufficient statistical power to analyze the associations among other variables of interest, such as cyst location and specific symptoms/findings.

\section{Conclusions}

Our exploratory analyses suggest that 3 groups of pediatric patients are more likely to undergo surgery for an intracranial arachnoid cyst, namely, patients whose cyst is large, compresses a narrow CSF flow pathway (e.g., cerebral aqueduct, foramina of Monro) to cause hydrocepha-

TABLE 4. Surgical follow-up and outcomes of all patients and per surgical technique

\begin{tabular}{|c|c|c|c|c|}
\hline \multirow[b]{2}{*}{ Outcome } & \multirow[b]{2}{*}{$\begin{array}{l}\text { All Cases } \\
(n=27)\end{array}$} & \multicolumn{3}{|c|}{ Surgical Technique } \\
\hline & & $\begin{array}{c}\text { Endoscopic } \\
\text { Fenestration }(n=7)\end{array}$ & CP Shunt $(n=10)$ & $\begin{array}{l}\text { Craniotomy-Based } \\
\text { Procedure }(n=7)\end{array}$ \\
\hline Follow-up time, mos (median [min, max]) & $61(1,207)$ & $21(1,107)$ & $75(23,207)$ & $21(7,126)$ \\
\hline Symptom resolution w/in 6 mos (no. [\%]) & $18(67)$ & $4(57)$ & $8(80)$ & $4(57)$ \\
\hline Need for reop to date (no. [\%]) & $11(41)$ & $3(43)$ & $5(50)$ & $2(29)$ \\
\hline$>1$ reop to date $($ no. $[\%])$ & $6(22)$ & $0(0)$ & $3(30)$ & $2(29)$ \\
\hline \multicolumn{5}{|l|}{ Complication (no. [\%]) } \\
\hline CSF leak & $5(19)$ & $1(14)$ & $1(10)$ & $3(43)$ \\
\hline Infection & $3(11)$ & $1(14)$ & $1(10)$ & $0(0)$ \\
\hline Hemorrhage & $1(4)$ & $1(14)$ & $0(0)$ & $0(0)$ \\
\hline \multicolumn{5}{|l|}{ Cyst-size change from before op } \\
\hline Median size, $\mathrm{cm}^{3}(\mathrm{IQR})$ & $-49(305)$ & $-51(115)$ & $-199(521)$ & $-87(39)$ \\
\hline Decrease in size $\left(>10 \mathrm{~cm}^{3}\right)$ (no. [\%]) & $18(67)$ & $5(71)$ & $7(70)$ & $6(86)$ \\
\hline Unchanged size (w/in $\left.10 \mathrm{~cm}^{3}\right)$ (no. [\%]) & $3(11)$ & $1(14)$ & $1(10)$ & $0(0)$ \\
\hline Increase in size $\left(>10 \mathrm{~cm}^{3}\right)($ no. $[\%])$ & $6(22)$ & $1(14)$ & $2(20)$ & $1(14)$ \\
\hline Length of stay, days (median [IQR]) & $5(3)$ & $3(1)$ & $5(1)$ & $5(6)$ \\
\hline
\end{tabular}

* Includes 2 patients with bur holes and 1 with a ventriculoperitoneal shunt. 
lus, or has ruptured/hemorrhaged. Furthermore, among patients who underwent surgery, there were no salient differences among the 3 surgical techniques (endoscopic fenestration, $\mathrm{CP}$ shunting, or craniotomy-based procedures) for several clinically important outcomes, including symptom resolution within 6 months, need for reoperation to date, cyst-size change from before the operation, morbidity, and mortality. However, given the limitations in current evidence, a larger prospective multicenter study is required to enable more robust analyses, which could ultimately provide a decision-making framework for surgical indications and clarify any differences in the comparative effectiveness of surgical approaches to treating pediatric intracranial arachnoid cysts.

\section{Acknowledgments}

We are grateful to Fidelma DiFlorio and Lisa van Oorschot for administrative assistance, Lorraine Adam-Doornbusch and Elviera Janzen for assistance with searching electronic medical records, Josie Pond for assistance with searching paper-based and archived records, and Eddy Malouf and Uttara Partap for critical feedback on an earlier version of the manuscript.

\section{References}

1. Al-Holou WN, Yew AY, Boomsaad ZE, Garton HJ, Muraszko KM, Maher CO: Prevalence and natural history of arachnoid cysts in children. J Neurosurg Pediatr 5:578-585, 2010

2. Alexiou GA, Varela M, Sfakianos G, Prodromou N: Shunting for the treatment of arachnoid cysts in children. Neurosurgery $67: 1632-1636,2010$

3. Ali ZS, Lang SS, Bakar D, Storm PB, Stein SC: Pediatric intracranial arachnoid cysts: comparative effectiveness of surgical treatment options. Childs Nerv Syst 30:461-469, 2014

4. Cagnoni G, Fonda C, Pancani S, Pampaloni A, Mugnaini L: [Intracranial arachnoid cyst in pediatric age.] Pediatr Med Chir 18:85-90, 1996 (Ital)

5. Cinalli G, Spennato P, Columbano L, Ruggiero C, Aliberti F, Trischitta V, et al: Neuroendoscopic treatment of arachnoid cysts of the quadrigeminal cistern: a series of 14 cases. J Neurosurg Pediatr 6:489-497, 2010

6. Cress M, Kestle JRW, Holubkov R, Riva-Cambrin J: Risk factors for pediatric arachnoid cyst rupture/hemorrhage: a case-control study. Neurosurgery 72:716-722, 2013

7. Go KG, Houthoff HJ, Blaauw EH, Havinga P, Hartsuiker J: Arachnoid cysts of the sylvian fissure. Evidence of fluid secretion. J Neurosurg 60:803-813, 1984

8. Guzel A, Tatli M, Kilincer C, Yilmaz F: Posttraumatic intraventricular arachnoid cyst accompanied by pseudomeningoencephalocele in a child. J Clin Neurosci 14:1210-1213, 2007

9. Harrell FE Jr, Lee KL, Mark DB: Multivariable prognostic models: issues in developing models, evaluating assumptions and adequacy, and measuring and reducing errors. Stat Med 15:361-387, 1996
10. Holst AV, Danielsen PL, Juhler M: Treatment options for intracranial arachnoid cysts: a retrospective study of 69 patients. Acta Neurochir Suppl 114:267-270, 2012

11. Kim BS, Illes J, Kaplan RT, Reiss A, Atlas SW: Incidental findings on pediatric MR images of the brain. AJNR Am J Neuroradiol 23:1674-1677, 2002

12. Morris Z, Whiteley WN, Longstreth WT Jr, Weber F, Lee YC, Tsushima Y, et al: Incidental findings on brain magnetic resonance imaging: systematic review and meta-analysis. BMJ 339:b3016, 2009

13. Peyser E, Weissberg D: Post-traumatic arachnoidal cyst. Report of an unusual case. J Neurosurg 18:551-553, 1961

14. Pradilla G, Jallo G: Arachnoid cysts: case series and review of the literature. Neurosurg Focus 22(2):E7, 2007

15. Rengachary SS, Watanabe I: Ultrastructure and pathogenesis of intracranial arachnoid cysts. J Neuropathol Exp Neurol 40:61-83, 1981

16. Schroeder HW, Gaab MR: Endoscopic observation of a slitvalve mechanism in a suprasellar prepontine arachnoid cyst: case report. Neurosurgery 40:198-200, 1997

17. Shim KW, Lee YH, Park EK, Park YS, Choi JU, Kim DS: Treatment option for arachnoid cysts. Childs Nerv Syst 25:1459-1466, 2009

18. Weber F, Knopf H: Incidental findings in magnetic resonance imaging of the brains of healthy young men. J Neurol Sci 240:81-84, 2006

19. Westermaier T, Schweitzer T, Ernestus RI: Arachnoid cysts. Adv Exp Med Biol 724:37-50, 2012

20. Zada G, Krieger MD, McNatt SA, Bowen I, McComb JG: Pathogenesis and treatment of intracranial arachnoid cysts in pediatric patients younger than 2 years of age. Neurosurg Focus 22(2):E1, 2007

\section{Author Contributions}

Conception and design: Singh, Ali, Farrokhyar. Acquisition of data: Singh, Ali, Bennardo, Zagzoog. Analysis and interpretation of data: Singh, Ali, Almenawer, Smith, Dao, Farrokhyar. Drafting the article: Ali. Critically revising the article: Singh, Ali, Bennardo, Almenawer, Zagzoog, Ajani, Farrokhyar. Reviewed submitted version of manuscript: all authors. Approved the final version of the manuscript on behalf of all authors: Singh. Statistical analysis: Ali, Smith, Dao, Farrokhyar. Administrative/technical/material support: Singh, Bennardo, Almenawer, Zagzoog, Smith, Dao, Ajani, Farrokhyar. Study supervision: Singh, Farrokhyar.

\section{Supplemental Information \\ Previous Presentation}

Portions of this work were presented in poster form at the 82nd Annual Scientific Meeting of the American Association of Neurological Surgeons held in San Francisco, California, on April 5-9, 2014.

\section{Correspondence}

Sheila K. Singh, McMaster Children's Hospital, Rm. 4E5, 1200 Main St. W, Hamilton, ON L8N 3Z5, Canada. email: ssingh@ momaster.ca. 